\section{Gastric Carcinoma with Discrete Intragastric and Duodenal Metastases: Endoscopic and Histological Findings}

We report here the case of a 56-year-old man who presented with mild mid-epigastric pain, weight loss, and intermittent black stools. Upper endoscopy revealed multiple "volcano-like" lesions with tip hemorrhage, involving the second portion of the duodenum (Figure 1) and the gastric cardia, and a deep ulcer in the prepyloric region. Biopsies obtained from the gastric cardia and duodenal lesions showed poorly differentiated carcinoma, while the biopsies from the prepyloric ulcer revealed carcinoma with a transitional zone from dysplastic epithelium to cancer cells (Figure 2). The biopsy was interpreted as adenocarcinoma, since the tumor cells were positive for anti-cytokeratin, negative for anti-S-100 on immunohistochemical studies, and both the mucin and PAS were all positive on histochemical examination-suggesting epithelial and glandular differentiation rather than sarcoma, germ-cell tumor, or melanoma. A diagnosis of primary adenocarcinoma of the stomach with discrete intragastric and duodenum metastases was established.

Metastatic tumors to the upper gastrointestinal tract have an overall prevalence of $1-4 \%$ in postmortem series (1). Metastatic tumors to the duodenum are very rare. Malignant melanoma, breast, and lung cancer are the most common primary lesions $(1-3)$. As far as we are aware, only three cases of gastric carcinoma with indirect metastatic duodenal involvement have been reported in the English literature since 1965 (4). The present rare case of prepyloric adenocarcinoma with discrete metastases in both directions, to the duodenum and to the gastric antrum and cardia, does not resemble any previous reports. Although hematogenous spread is possible in this case, the pathway of dissemination is not well understood. Although no single endoscopic appearance is characteristic of any specific cancer, a finding of many "volcano-like" lesions of varying sizes in the upper gastrointestinal tract is most likely to be due to metastatic tumors, as in our case. Other differential diagnoses with "volcano-like" lesions in the upper gastrointestinal tract include spindle-cell tumors, aberrant pancreas, carcinoid tumor, primary carcinoma, eosinophilic granuloma, Kaposi’s sarcoma, lymphoma, submucosal hemorrhage, and gastric ulcers.

T.-H. Young', Y.-C. Chao' ', H.-S. Tang', C.-T. Hsu', H.-S. Lee' , C. Yeh'

' Division of Gastroenterology, Dept, of Internal Medicine

2 Dept. of Pathology, Tri-Service General Hospital, National

Defense Medical Center, Taipei, Taiwan

\section{References}

1. Telerman A, Gerard B, Van den Heule B, et al. Gastrointestinal metastases from extra-abdominal tumors. Endoscopy 1985; 17: 99- 101

2. Nelson RS, Lanza F. Malignant melanoma metastatic to the upper gastrointestinal tract: endoscopic and radiologic correlations form and evolution of lesions and value of directed biopsy in diagnosis. Gastrointest Endose 1978; 24: 156-8.

3. Hartman W, Sherlock P. Gastroduodenal metastases from carcinoma of the breast. Cancer 1961: 14:426-31.

4. Raijman I, Catalano M, Sellin J, et al. Gastric carcinoma spread to the duodenum. Gastrointest Endose 1991: 37: 556-9.

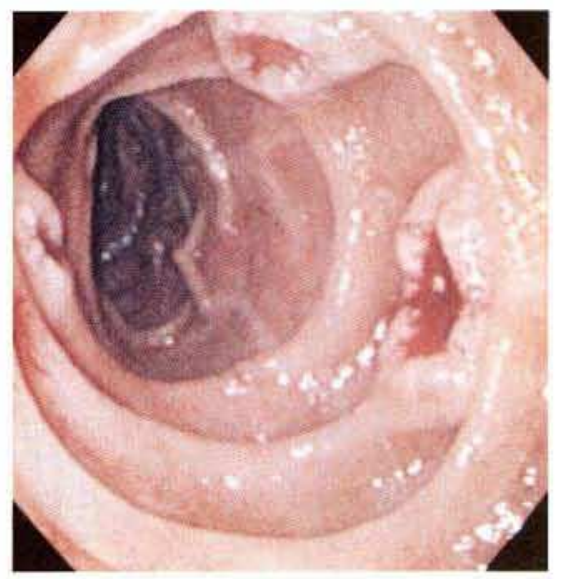

Figure 1: Endoscopic view of the second portion of the duodenum, showing three "volcano-like" lesions of varying sizes, with tip hemorrhage and normallooking intervening mucosa

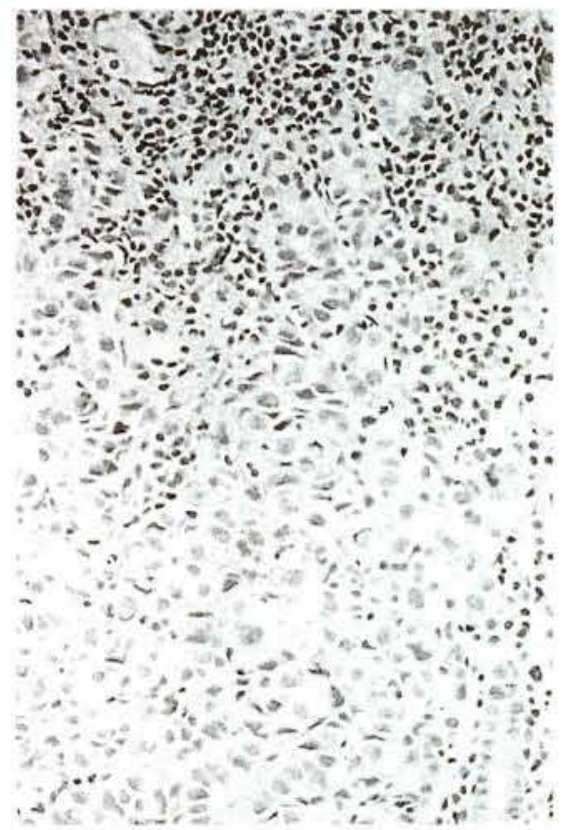

Figure 2: The histological specimen of the prepyloric ulceration reveals a poorly differentiated adeno carcinoma with a transitional zone from dys. plastic epithelium to cancer cells (hematoxylin-eosin, $\times 200$ )
Corresponding Author

C. Yeh, M.D.

Division of Gastroenterology

Dept, of Internal Medicine

Tri-Service General Hospital

40 Sec, 3

Ting-Chow Road

Taipei

Taiwan

Fax: +11-886-2-3657292 